\title{
¿CITAN LAS MUJERES INVESTIGADORAS MÁS A LAS OTRAS MUJERES QUE A LOS HOMBRES?
}

\author{
Nicoleta-Roxana Dinu* \\ https://orcid.org/0000-0002-1150-3469
}

RECIBIDO: Febrero 2021 / ACEPTADO: Mayo 2021 / PUBLICADO: Septiembre 2021

Como citar: Dinu, Nicoleta-Roxana. (2021) ¿Citan las mujeres investigadoras más a las otras mujeres que a los hombres? Telos: revista de Estudios Interdisciplinarios en Ciencias Sociales, 23 (3), Venezuela. (Pp. 568-583).

DOI: www.doi.org/10.36390/telos 233.05

\section{RESUMEN}

El objetivo de la investigación presentada en este artículo es analizar posibles sesgos de citación entre artículos científicos debidos al género de los autores. Diversos investigadores, como Maliniak, Powers y Walter (2013); Beaudry y Larivière (2016); Ghiasi et al. (2018); Huang et al. (2019); Potthoff y Zimmermann (2017); y Thelwall (2020), han puesto de relieve la brecha de género existente en las carreras universitarias, y también en la publicación científica, reflejo de lo que ocurre en la Sociedad en general. La hipótesis de partida de esta investigación es que debido a la discriminación de que son objeto, las mujeres podrían reaccionar citando más a otras autoras. La metodología usada ha sido 1) la realización de una búsqueda bibliográfica sobre el tema. Los resultados de la búsqueda, que expresan situaciones negativas para las mujeres, sirven como preámbulo del razonamiento que se lleva a cabo sobre posibles reacciones de las mujeres ante tales situaciones, y del planteamiento de esta investigación, preguntándonos si de forma consciente o por sensibilidad y afinidad las mujeres tienen alguna tendencia a citar más los trabajos de sus congéneres que los de los colegas masculinos. 2) Se han seleccionado los artículos de dos números completos de cuatro revistas, y se han contabilizado las citas de hombres a hombres, hombres a mujeres, mujeres a mujeres y mujeres a hombres. Los resultados obtenidos muestran una diferencia de comportamiento según el género: las mujeres son más equilibradas: citan un $40 \%$ a mujeres y un $60 \%$ a hombres. En cambio, los hombres demuestran más homofilia enviando solo un $25 \%$ de citas a las mujeres y un $75 \%$ a sus congéneres. Se concluye que perdura la brecha de género y que continúa una situación perjudicial para las mujeres que, al no recibir la merecida acreditación por sus trabajos, no consiguen alcanzar los niveles superiores de las carreras académicas.

\footnotetext{
* Doctora en Filología por la Universidad de Bucarest. Trabaja en la Biblioteca Nacional de Rumanía, Departamento de Desarrollo Institucional. Correo electrónico: roxana.dinu@bibnat.ro, roxadinu@gmail.com
} 
Palabras clave: Brecha de género; Hombres; Mujeres; Carrera académica; Publicación científica; Revistas; Citas; Citaciones; Hábitos; Homofilia masculina; Acreditación.

\section{Do women researchers cite other women more than men?}

\section{ABSTRACT}

The objective of the research presented in this article is to analyze possible citation biases between scientific articles due to the gender of the authors. Various researchers, such as Maliniak, Powers, and Walter (2013); Beaudry and Larivière (2016); Ghiasi et al. (2018); Huang et al. (2019); Potthoff and Zimmermann (2017); and Thelwall (2020), have highlighted the existing gender gap in university careers, and also in scientific publication, a reflection of what happens in society in general. The starting hypothesis of this research is that due to the discrimination they are subjected to, women could react by citing other female authors more. The methodology used has been 1) conducting a bibliographic search on the subject. The search results that express negative situations for women serve as a preamble to the reasoning that is carried out about possible reactions of women to such situations, and the approach of this research, asking us if consciously or out of sensitivity and affinity, women have some tendency to cite the work of their peers more than those of male colleagues. 2) Articles from two complete issues of four journals have been selected, and citations from men to men, men to women, women to women, and women to men have been counted. The results obtained show a difference in behavior according to gender: women are more balanced $40 \%$ cite women, and $60 \%$ cite men. In contrast, men show more homophilia, sending only $25 \%$ of citations to women and $75 \%$ to their peers. It is concluded that the gender gap persists and that a detrimental situation continues for women who, by not receiving the deserved accreditation for their work, are unable to reach the higher levels of academic careers.

Keywords: gender gap; men; women; academic career; scientific publication; scholarly publication; journals; cites; citations; habits; male homophilia; accreditation.

\section{Introducción}

Las diferencias de género, en las que usualmente las mujeres salen perjudicadas respecto de los hombres han sido estudiadas desde diferentes situaciones y perspectivas. Así por ejemplo Alvarado-López y Martín-García (2020), hallaron que subsisten las diferencias entre el número de mujeres y hombres que trabajan en todos los departamentos de las agencias de publicidad y que en España la Ley de igualdad de género no parece haber tenido efectos visibles en relación con los techos de cristal que el sector impone a las profesionales.

Arzuaga-Williams $(2020$, p. 6$)$ halló que:

Los profesionales varones de la Comunicación Organizacional en Uruguay, más que las profesionales mujeres, deciden sobre políticas o planes de largo plazo, toman decisiones que implican recursos muy variados (económicos, humanos, tecnológicos) que pueden afectar varios sectores de la organización, y son los responsables de los resultados de la comunicación, sean positivos o negativos. Es decir, los varones desempeñan el rol decisor en Comunicación Organizacional en mayor proporción que las mujeres. 
Se podría suponer que en el entorno universitario, la brecha de género es menor que en las empresas, sin embargo Etura-Hernández, Martín-Jiménez y Ballesteros-Herencia (2019), considerando que la educación es uno de los pilares fundamentales en la socialización de las personas, estudiaron las experiencias, opiniones, y distorsiones de la comunidad universitaria respecto a la igualdad y la violencia de género. Detectaron que el $50 \%$ de los encuestados definía erróneamente la violencia de género y desconocía las políticas de igualdad de la universidad donde se llevó a cabo la encuesta, aunque la mayoría se mostró a favor de la implicación de la Institución en la educación, formación y sensibilización de la comunidad universitaria en materia de igualdad.

Las diferencias entre hombres y mujeres en la ciencia son un reflejo y una consecuencia de las diferencias que se producen en todos los aspectos de la vida humana. Hay muchas causas reales que contribuyen a la situación actual, biológicas, sociales (Brown; Samuels, 2018), psicológicas, pero también algunos prejuicios.

En la siguiente sección 2 se presentan los resultados de una búsqueda bibliográfica sobre las diferencias de género que se producen en la Sociedad. Estos resultados, que en su gran mayoría muestran situaciones perjudiciales para las mujeres, han sido los preliminares de este trabajo. En la sección 3 se explican los principales objetivos perseguidos en esta investigación, en la 4 la metodología, y en la 5 se presentan los resultados. El artículo finaliza con una discusión de éstos, y unas conclusiones, incluyendo las limitaciones del estudio y señalando futuras líneas de investigación.

\section{Principales estudios sobre brecha de género en el entorno académico}

Uno de los primeros estudios sobre las diferencias de género en la ciencia fue el de Larivière et al. (2013), en el que indican que las mujeres publican significativamente menos artículos sobre todo en áreas en las que la investigación es costosa, como la física de altas energías, "posiblemente como resultado de políticas y procedimientos relacionados con la asignación de fondos". Las mujeres participan menos en proyectos que conducen a la publicación y es poco probable que figuren como primer autor de los artículos.

Fox, Whittington y Linkova (2017, p. 27) señalan que:

(...) las jerarquías de género en las carreras científicas reflejan y refuerzan las relaciones de estatus y poder en las sociedades. A pesar del aumento del número de mujeres en ciencia en las últimas tres décadas, hemos observado una disparidad de género profunda y persistente en la participación por campo y en el desempeño, el reconocimiento y las recompensas en las carreras científicas. Las explicaciones de estas disparidades no radican simplemente en los intereses individuales, las intenciones y las opciones de mujeres y hombres para seguir una educación y carreras científicas, sino a influencias institucionales complejas, incluido el matrimonio, la familia y el hogar, y en la cultura y las prácticas que conectan la ciencia y la masculinidad.

González-Alcaide y González-Teruel (2020), estudian la dirección de tesis doctorales en Biblioteconomía y Documentación en las universidades española y hallan que, aunque en conjunto el número de mujeres $(51,7 \%)$ que dirigen tesis es superior al de los hombres $(48,3 \%)$, luego su presencia va reduciéndose a medida que aumenta el número de direcciones. Observan 
que las mujeres participan de forma mucho más reducida en las tesis dirigidas por dos o más codirectores, con únicamente un $38,5 \%(n=231)$ de codirecciones de mujeres frente al $61,5 \%$ $(n=369)$ en el caso de los hombres. También las mujeres ocupan posiciones subordinadas, con una presencia mucho menor como primeras codirectoras ( $41,3 \%$ de mujeres frente al $61,7 \%$ de hombres en las tesis con dos codirecciones; y $25 \%$ de mujeres frente al $75 \%$ de hombres en el caso de las tesis con 3 codirecciones).

Brown y Samuels (2018); y Dion, Sumner y Mitchell (2018), comentan cómo el hecho de que las mujeres publiquen menos, hace que sus carreras se resientan, y acaben teniendo menos presencia e impacto en su campo de actividad.

Según los citados Dion, Sumner y Mitchell (2018) la brecha de género de citas se reduce cuando existen más mujeres publicando en una disciplina, y no existe tal brecha en los artículos escritos por mujeres (algo que pretendemos corroborar en este artículo).

Jordan Dworkin y sus colegas (2020), comentado por Budrikis (2020), presentan un estudio de caso de patrones de citas en las principales revistas de neurociencia. Encontraron que los artículos cuyos primeros y últimos autores (en algunas disciplinas acostumbran a firmar en último lugar los investigadores senior) son hombres están $23,4 \%$ sobrerrepresentados en las listas de referencias, y que la brecha de género está empeorando a razón de $0,4 \%$ cada año. En la misma línea, Fulvio, Akinnola y Postle (2020) hallaron que los artículos escritos por hombres como primer y último autor estuvieron sobrecitados en comparación con lo que se esperaría en base al número de artículos publicados por la revista. Por el contrario, los artículos escritos por equipos con al menos una mujer en la posición de primer o último autor fueron subcitados.

En un estudio longitudinal de los años 1984, 1994, 2004 y 2014, Huang et al. (2019), encuentran que los artículos cuyo primer autor es una mujer reciben menos citas, pero la brecha se va reduciendo pasando de un $26,1 \%$ en 1984 a 10,3\% en 2014 .

A pesar de los avances recientes en igualdad, es probable que la brecha de género persista durante generaciones, particularmente en cirugía, informática, física y matemáticas (Holman; Stuart-Fox; Hauser, 2018, pp. 4 y 9), y se hace mayor a medida que se avanza en la carrera. Por ello, --según estos autores-- las revistas de prestigio publican menos artículos de escritoras, y estiman que tales revistas invitan a presentar artículos dos veces más a los hombres que a las mujeres. Los países ricos, en particular Japón, Alemania y Suiza, tienen menor proporción de escritoras que los más pobres. La brecha de género no se cerrará sin no se introducen reformas en educación, investigación y publicación (Holman; Stuart-Fox; Hauser, 2018, p. 1). El avance de partidos políticos de ultraderecha no favorece la igualdad de género (Ballesteros-Herencia y Gómez-García, 2020, p. 7).

Ghiasi et al. (2018), señalan que la homofilia de género en las citas existe en todas las disciplinas, pero las tasas desproporcionadas de autocitas de los autores masculinos incrementan las disparidades. La mayor tendencia de los hombres a citar el trabajo de sus pares masculinos podría hacer que las contribuciones científicas de las mujeres sean menos reconocidas. Los resultados de su estudio cuestionan la neutralidad de las métricas basadas en citas y, por lo tanto, su uso como instrumentos de evaluación en el sistema de acreditación académica.

Investigando un aspecto curioso relacionado, Krawczyk (2017), examinó la bibliografía académica en busca de casos de atribución incorrecta del género del autor citado. Si bien estos 
errores no son en general comunes, su frecuencia depende acusadamente del género del autor citado: las investigadoras son citadas como si fueran hombres más de diez veces más a menudo de lo que sucede al contrario, lo que probablemente revela que las citas están influenciadas por el estereotipo de género en la ciencia. El género del autor citante y la disciplina parecen tener poco efecto (contrariamente a lo señalado por Thelwall, 2020).

Algunos trabajos han estudiado el nivel de autocitación de hombres y mujeres. La autocitación a menudo se interpreta como un acto de autopromoción que (artificialmente) aumenta la visibilidad del trabajo previo del autor a corto plazo, lo que luego podría inflar la autoridad profesional a largo plazo (Azoulay; Lynn, 2020). Las comparaciones partieron de la hipótesis de considerar los hombres más agresivos en las acciones de promoción, lo que incluiría autocitarse más, ello a pesar de que las mujeres se relacionan más que los hombres en las redes sociales (López-de-Ayala; Vizcaíno-Laorga; Montes-Vozmediano, 2020). También, algunos investigadores han demostrado que las mujeres evalúan su desempeño de manera más crítica que los hombres (Correll, 2004; Exley y Kessler, 2019), incluso tienden a caracterizarse retrospectivamente por haber tenido un desempeño peor que el resultado real, mientras que los hombres tienden a recordarse ellos mismos como habiendo hecho algo mejor de lo que realmente fue (Reuben et al., 2014). De forma similar, Herbst (2020, p. 7) halló que

(...) las mujeres tienen una mayor tendencia a subestimar sus habilidades 0 desempeño al no atribuirse el mérito del éxito y atribuir su éxito más a fuentes externas que a su capacidad.

King et al. (2017) y Mishra et al. (2018), llegaron a resultados contradictorios sobre si los hombres se citan más o menos que las mujeres, y en un tercer estudio Azoulay y Lynn (2020), no hallaron diferencias. Igualmente, Andersen y Nielsen (2018), esperaban ver un importante sesgo masculino en la WoS, en comparación con el más inclusivo Google Scholar, derivado del hecho que que las mujeres ven truncadas o dificultadas sus carreras por su mayor dedicación a la familia, pero hallaron que dicho sesgo de género se percibe en Química y Cirugía, pero es marginal en ciencias sociales.

Beaudry y Larivière hallaron que:

(...) cuando las mujeres colaboran con el mismo número de coautores que los hombres, o publican en revistas de factor de impacto similares, sus artículos se citan menos que los de sus colegas masculinos. [...] Nuestros resultados muestran, de manera preocupante, que los académicos que publican con una proporción mayor de coautoras son menos citados. Además, cuando publican en revistas de factor de impacto similares, los investigadores que colaboran con una mayor proporción de coautores mujeres son consistentemente menos citados en los campos de la salud que si estuvieran publicando con un grupo de coautores dominado por hombres (Beaudry; Larivière, 2016, p. 1790).

Benjamens et al. (2020): corroboraron los resultados anteriores con respecto a las diferencias en las tasas de citas entre autores masculinos y femeninos. Las autoras están especialmente subrepresentadas en las publicaciones muy citadas (por ej., las que reciben más de 200 citas). Estos autores piden empoderar a las mujeres en la ciencia, crear conciencia social, nivelar las demandas de estilo de vida entre hombres y mujeres (que es la razón de fondo de 
las diferencias), y que las revistas científicas y los comités científicos aumenten la representación de las mujeres, al menos para compensar en parte su dedicación a la maternidad.

Lerchenmueller et al. (2019), proporcionan una explicación parcial del predominio de los autores masculinos con respecto a las tasas de citación. Con un análisis bibliométrico extenso, este estudio mostró que los autores masculinos presentan sus resultados con términos más positivos y afirmaciones más fuertes, lo que da por resultado tasas de citas más altas. En general, la disparidad en las tasas de citas se puede explicar por las diferencias en la presentación de los resultados de la investigación, promoción y subrepresentación de mujeres en consorcios de investigación de alto impacto.

En un estudio dentro de la disciplina de relaciones internacionales (RI) Maliniak, Powers y Walter (2013), también demostraron que las mujeres son citadas sistemáticamente menos que los hombres. Después de un análisis de redes para investigar hasta qué punto el género del autor de un artículo afecta la centralidad relativa de ese artículo en la red de citas, hallaron que, en igualdad de condiciones, los artículos escritos por mujeres son sistemáticamente menos centrales que los artículos escritos por hombres. Creen que las causas pueden ser dos: 1) las mujeres tienden a citarse menos que los hombres, y 2) los hombres (que son mucho más numerosos en el campo de RI) tienden a citar a sus colegas masculinos que a las mujeres. La situación influye en el avance académico de estas, pues los recuentos de citas se utilizan como una medida clave de la calidad y el impacto de la investigación.

Potthoff y Zimmermann (2017), comentan que varios estudios informan que los académicos varones citan publicaciones de autores masculinos con más frecuencia que sus colegas femeninas, y también que las mujeres citan más a mujeres. Esta homofilia de género en las citas podría apuntar a una fragmentación de la ciencia debida al género.

Nunkoo et al. (2019), exploran las brechas de género y las diferencias en las prácticas de citación de los académicos en los artículos más citados en la investigación turística. Los resultados sugieren que los investigadores masculinos dominan la autoría de esos artículos y es más probable que se autociten que las mujeres. El estudio también encuentra una disparidad en el recuento de citas entre los artículos escritos por hombres y mujeres. Controlando otros factores, el género del autor es un determinante importante del recuento de citas.

En cambio, Nielsen (2017), en un estudio del campo de la gestión y la administración encontró incluso una pequeña diferencia a favor de las citas de mujeres, cosa que concordaba con que hay una mayoría de mujeres entre el $10 \%$ de artículos más citados en la citada disciplina.

Igualmente, Thelwall (2020), en un amplio estudio halló que las diferencias de género en el impacto de las citas de artículos de revistas varían por países y disciplinas, siendo positivas en unos países y negativas en otros. Las diferencias de género oscilan según el año, y no pudo apreciar una tendencia: ni aumenta ni disminuye con el tiempo. En promedio el efecto es pequeño y es poco probable que tenga una influencia sustancial en las carreras de los investigadores.

Dion, Sumner y Mitchell (2018), hallaron que en algunas áreas concretas dentro de las ciencias políticas y las ciencias sociales en las que las mujeres participan más, éstas tienen brechas de citas más pequeñas: se produce una reducción del "efecto Mateo" que ocurre en áreas dominadas por los hombres. Sin embargo, las brechas pueden persistir si ocurren "efectos Matilda"1 en el que la investigación de las mujeres se considera menos importante o sus ideas 
se atribuyen a académicos varones. Estos autores también observaron una subcitación del trabajo de las mujeres, incluso en revistas en las que publican en su mayoría autoras femeninas.

En el espacio iberoamericano encontramos el interesante estudio de Torres-Salinas, Muñoz-Muñoz y Jiménez-Contreras (2011), sobre las áreas de Ciencias Sociales y Jurídicas:

(...) existen diferencias significativas en la capacidad de producción de trabajos, ya que las mujeres en todas las áreas analizadas presentan un menor número de trabajos que los hombres. En citas también las mujeres obtienen valores inferiores y sólo en dos áreas consiguen superar a los hombres. Sin embargo, esta situación se invierte en el caso de las citas internacionales, ya que en el $64 \%$ de las áreas analizadas las mujeres presentan valores superiores. El promedio de citas alcanzado por las mujeres es mayor en todas las áreas comparadas con el promedio de hombres, exceptuando el área de Biblioteconomía.

Según los citados autores, las mujeres producen menos trabajos que los hombres, pero reciben más citas/documento: los trabajos publicados por las investigadoras son citados con mayor frecuencia que los de los hombres, lo que indica que producen documentos de mayor calidad (Torres-Salinas; Muñoz-Muñoz; Jiménez-Contreras, 2011).

Como síntesis de nuestra revisión bibliográfica se puede decir que en general las mujeres salen perjudicadas en la mayoría de los casos estudiados, pero en algunas disciplinas en las que son más prolíficas, la situación del reconocimiento a su trabajo está equilibrada (Thelwall, 2020; Torres-Salinas; Muñoz-Muñoz; Jiménez-Contreras) o incluso está a su favor (Nielsen, 2017).

\section{Objetivo de esta investigación}

Iranzo-Cabrera (2020), analiza la gestación y consolidación del activismo feminista que ha aglutinado a miles de profesionales de la comunicación en España a raíz de la huelga del 8 de marzo de 2018, unidas a través de la aplicación de mensajería Telegram y por el hashtag \#lasperiodistasparamos en Twitter.

A raíz de un sentimiento de empoderamiento surgido del hartazgo, la indignación, la superación del miedo y la denuncia compartida ante la opinión pública de actitudes y perspectivas machistas en su profesión: brecha salarial, techo de cristal, dinámicas de trabajo basadas en el presentismo y la libre disposición, acoso sexual y laboral, espacios de opinión masculinizados y enfoque androcéntrico de los temas, tal y como denuncian en su manifiesto.

En esta investigación que se realiza en el campo de la Comunicación y la Documentación se quiere conocer si la generalizada subcitación de los trabajos de las mujeres respecto de la de los hombres ha producido una reacción para invertir el fenómeno. ¿Es que en esta área las mujeres citan más a las mujeres, y los hombres más a los hombres, como hallaron Potthoff y Zimmermann (2017)?

Para ello se establece la siguiente hipótesis:

$\mathrm{H}$. Conscientes de las varias brechas de género y techos de cristal que perjudican a las mujeres, las mujeres son más sensibles 0 afines a las tesis e investigaciones de otras mujeres, y por ello tienen más en cuenta sus trabajos que los de los hombres. Según esto, ellas tomarían medidas solidarias forzando la lectura y la consiguiente citación de trabajos firmados por otras mujeres. 


\section{Metodología \\ Búsqueda bibliográfica}

Con el fin de estudiar el state-of-the-art de este tema, en enero de 2021 se realizaron búsquedas bibliográficas en las bases de datos Web of Science, Scopus y Google Scholar combinando los dos conceptos básicos 'mujer' y 'citaciones' definidos, respectivamente, con estos dos conjuntos de términos:

woman + women + mujer ${ }^{*}+$ gender + género* + sex + sexo + female + femenino + feminist ${ }^{\star}$ y citations + citaciones + cites + citas + ciencia + science + scientific $^{*}+$ cientific $^{*}+$ research ${ }^{*}+$ investiga $^{*}+$ scholar $^{*}+$ academic $^{*}+$ authorship + autoría

Para evitar excesivo ruido documental los resultados se limitaron a las referencias en las que las parejas de palabras aparecían en los títulos.

La búsqueda más precisa para los fines de este trabajo se obtuvo con las palabras "citations" y "citaciones", pero también se han encontrado artículos interesantes combinando las otras palabras listadas arriba. En una primera fase se seleccionaron 229 trabajos según se estimó su pertinencia a partir de los títulos. A leer los correspondientes resúmenes se seleccionó un conjunto de 38 trabajos que son los que se comentan y se citan a lo largo de este texto, y se listan en la sección final de Referencias.

\section{Obtención de los datos de la investigación}

Con el fin de comprobar el comportamiento de los autores femeninos y masculinos con respecto al uso y citación de los trabajos que sirven de base a sus respectivas investigaciones en Ciencias Sociales, se eligieron 4 conocidas revistas, se seleccionaron al azar dos de sus números de 2020 y se examinaron las referencias bibliográficas citadas en cada uno de sus artículos:

Comunicar (España), indexada en Scopus y WoS (SSCl).

https://www.revistacomunicar.com

Vol. XXVIII, núm. 62, 1r trimestre 2020

Vol. XXVIII, núm. 63, $2^{\circ}$ trimestre 2020

Profesional de la información (EPI) (España), indexada en Scopus y WoS (SSCl). http://profesionaldelainformacion.com/index.html

Vol. 29, núm. 2, marzo-abril de 2020

Vol. 29, núm. 3, mayo-junio de 2020

Revista Española de Documentación Científica (REDC) (España), indexada en Scopus y WoS (SSCl).

http://redc.revistas.csic.es/index.php/redc

Vol. 43, núm. 1, enero-marzo de 2020

Vol. 43, núm. 2, abril-junio de 2020 
Telos. Revista de Estudios Interdisciplinarios en Ciencias Sociales (Venezuela), indexada en WoS (ESCl).

http://ojs.urbe.edu/index.php/telos

Vol. 22, núm. 1, enero-abril de 2020

Vol. 22, núm. 2, mayo-agosto de 2020

Las 3 primeras revistas se eligieron porque son muy conocidas y consolidadas, especializadas en Información y Comunicación, la disciplina que se pretendía estudiar, con una publicación regular desde hace muchos años (28, 29 y 43 respectivamente) y están indexadas en las bases de datos más selectivas, por lo que sin duda son las más representativas del área. La cuarta revista, Telos, se añadió en principio porque era nuestra revista objetivo donde pretendíamos publicar, pero sobre todo porque publica también sobre Información y Comunicación, y está abierta a otras temáticas de Ciencias Sociales. El comportamiento bastante similar a las otras tres en cuanto patrones de citación nos hizo pensar que habíamos obtenido ya una muestra suficiente para nuestro propósito, y que añadir más revistas no cambiaba de forma importante los resultados.

Se seleccionaron solo los artículos firmados exclusivamente por un autor o por varios autores del mismo sexo, es decir, se descartaron los que tenían autoría mixta, al objeto de simplificar los cálculos y tener unos resultados más contrastados y nítidos. Hubiera sido complicado considerar autorías mixtas y fraccionar la influencia de cada autora o de cada autor. Se contabilizaron cuántas citas dirigían los artículos seleccionados a los trabajos citados en las referencias, y así mismo, sólo se tuvieron en cuenta los que tenían un sólo autor o varios del mismo género. Se excluyeron las autocitas.

En las revistas EPI y Telos fue fácil determinar el género de los autores, porque sus nombres están explícitos, aunque se tuvo dificultad para distinguir el sexo de algunos autores escandinavos, y sobre todo asiáticos e indios; tampoco fue fácil con algunos hispanos de América Latina. Sin embargo, en las referencias citadas de Comunicar y REDC los nombres están abreviados a la inicial y supuso más tiempo de investigación de cada una.

\section{Resultados}

La tabla 1 presenta los resultados obtenidos. Casualmente en la muestra estudiada de 58 artículos, exactamente la mitad estaban firmados exclusivamente por una o varias mujeres, y la otra mitad por uno o varios hombres.

En las filas se sitúan las cuatro revistas, con las autorías de solo mujeres y de solo hombres. $Y$ en las columnas el número de artículos que citan solo a mujeres y solo a hombres.

Tabla 1. Citas de autores femeninos y masculinos a otros autores del mismo sexo y de sexo contrario

\begin{tabular}{|c|c|c|c|c|c|c|}
\hline Autores & $\begin{array}{c}\text { N. de } \\
\text { artículos }\end{array}$ & $\begin{array}{l}\text { Total } \\
\text { citas }\end{array}$ & $\begin{array}{c}\text { Citas a } \\
\text { mujeres }\end{array}$ & $\%$ & $\begin{array}{c}\text { Citas a } \\
\text { hombres }\end{array}$ & $\%$ \\
\hline \multicolumn{7}{|l|}{ Comunicar } \\
\hline $\begin{array}{l}\text { Autoría de } \\
\text { solo } \\
\text { mujeres }\end{array}$ & 4 & 88 & 30 & 34,09 & 58 & 65,91 \\
\hline
\end{tabular}


Dinu

Vol. 23 (3): 568-583. Septiembre-Diciembre 2021

\begin{tabular}{|c|c|c|c|c|c|c|}
\hline $\begin{array}{l}\text { Autoría de } \\
\text { solo } \\
\text { hombres }\end{array}$ & 2 & 74 & 17 & 22,97 & 57 & 77,03 \\
\hline \multicolumn{7}{|c|}{ Profesional de la información (EPI) } \\
\hline $\begin{array}{l}\text { Autoría de } \\
\text { solo } \\
\text { mujeres }\end{array}$ & 18 & 485 & 233 & 48,04 & 252 & 51,96 \\
\hline $\begin{array}{l}\text { Solo } \\
\text { hombres }\end{array}$ & 15 & 376 & 101 & 26,86 & 275 & 73,14 \\
\hline \multicolumn{7}{|c|}{ Revista española de documentación científica (REDC) } \\
\hline $\begin{array}{l}\text { Autoría de } \\
\text { solo } \\
\text { mujeres }\end{array}$ & 1 & 51 & 14 & 27,45 & 37 & 72,55 \\
\hline $\begin{array}{l}\text { Autoría de } \\
\text { solo } \\
\text { hombres }\end{array}$ & 2 & 45 & 12 & 26,67 & 33 & 73,34 \\
\hline \multicolumn{7}{|l|}{ Telos } \\
\hline $\begin{array}{l}\text { Autoría de } \\
\text { solo } \\
\text { mujeres }\end{array}$ & 6 & 111 & 19 & 17,12 & 92 & 82,88 \\
\hline $\begin{array}{l}\text { Autoría de } \\
\text { solo } \\
\text { hombres }\end{array}$ & 10 & 178 & 42 & 23,6 & 136 & 76,40 \\
\hline \multicolumn{7}{|c|}{ Total de las 4 revistas } \\
\hline Autores & $\begin{array}{c}\text { N. de } \\
\text { artículos }\end{array}$ & $\begin{array}{l}\text { Total } \\
\text { citas } \\
\end{array}$ & $\begin{array}{c}\text { Citas a } \\
\text { mujeres }\end{array}$ & $\%$ & $\begin{array}{c}\text { Citas a } \\
\text { hombres }\end{array}$ & $\%$ \\
\hline $\begin{array}{l}\text { Autoría de } \\
\text { solo } \\
\text { mujeres }\end{array}$ & 29 & 735 & 296 & 40,27 & 439 & 59,73 \\
\hline $\begin{array}{l}\text { Autoría de } \\
\text { solo } \\
\text { hombres }\end{array}$ & 29 & 673 & 172 & 25,56 & 501 & 74,44 \\
\hline
\end{tabular}

Fuente: Elaboración propia.

Los datos de la tabla 1 se han representado en los gráficos 1 (citas emitidas por autoras) y 2 (citas emitidas por autores), que permiten ver mejor las diferencias de comportamiento. 
Gráfico 1. Citas de mujeres a mujeres y a hombres.

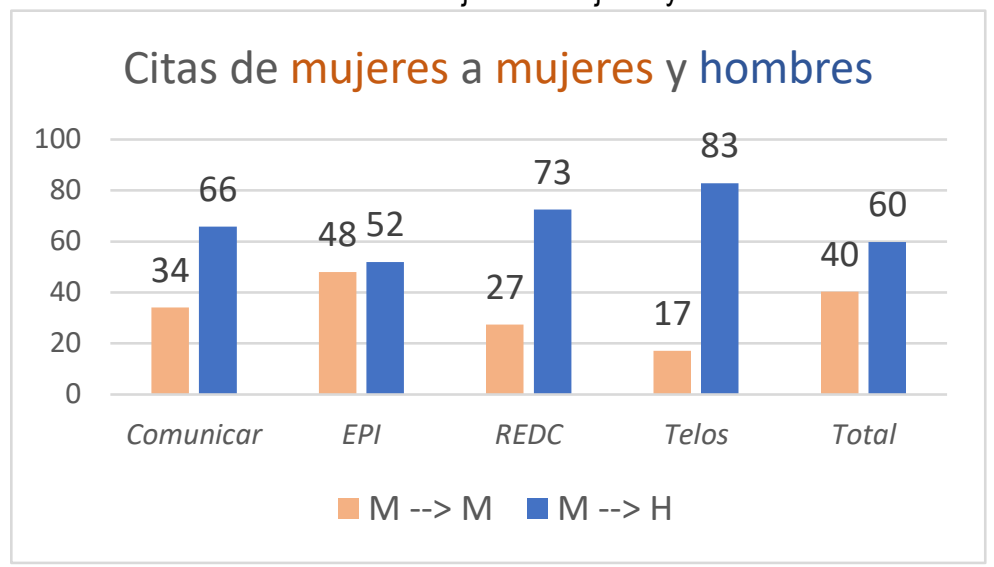

Fuente: Elaboración propia.

En el gráfico 1 se observa que en todas las revistas las autoras citan más a los autores masculinos que a sus congéneres. La diferencia mayor la encontramos en Telos y la menor en EPI. En conjunto, como promedio, las mujeres citan un $60 \%$ de trabajos masculinos y un $40 \%$ de femeninos.

En cambio con los autores masculinos se produce una homofilia -similar en las 4 revistas-: los hombres citan $75 \%$ de trabajos realizados exclusivamente por hombres y sólo un $25 \%$ de trabajos realizados por mujeres.

Gráfico 2. Citas de hombres a mujeres y a hombres.

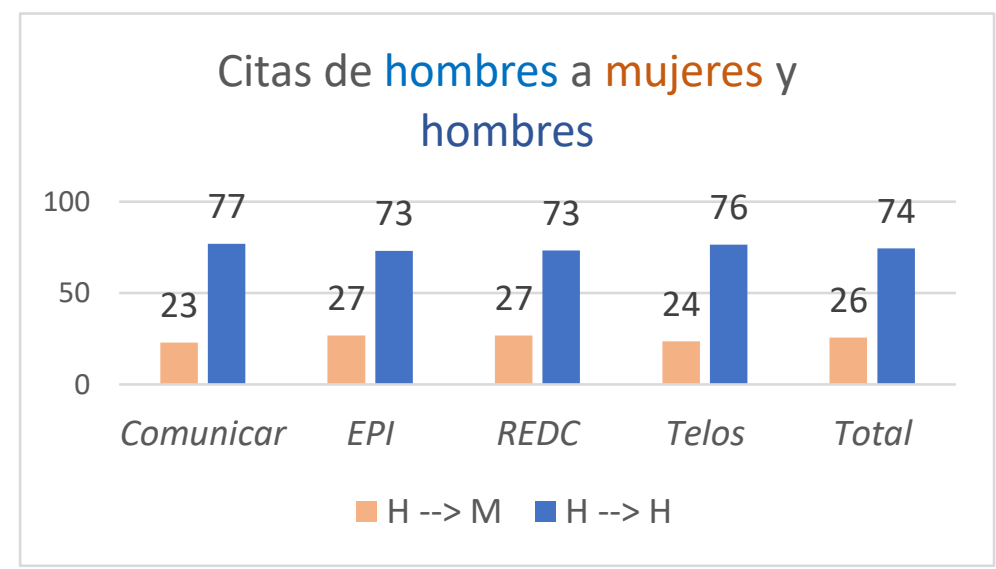

Fuente: Elaboración propia. 


\section{Discusión y conclusiones}

Los resultados demuestran que aparentemente por parte de las mujeres no se produce una fragmentación debida al género, como sí hallaron Potthoff y Zimmermann (2017), es decir no se observa una mayor citación de las autoras a sus colegas femeninos.

Así pues, nuestros resultados refutan la hipótesis de partida expresada al final de la sección 3, por lo que podemos afirmar que:

"las mujeres no son más sensibles 0 afines a las tesis e investigaciones de otras mujeres, y no tienen más en cuenta sus trabajos que los de los hombres. Ellas no toman medidas solidarias forzando la lectura y la consiguiente citación de trabajos firmados por otras mujeres"

Al contrario, las mujeres citan más los trabajos de los hombres. No obstante, las cifras pueden ocultar casos aislados de autoras que ya sea por afinidad o por reivindicación tengan preferencia en basar sus investigaciones en trabajos de otras investigadoras. Para conocer mejor esas actitudes y los comportamientos deberían hacerse entrevistas con cierta profundidad a autoras para discernir si conscientemente tienen en cuenta el género a la hora de seleccionar bibliografía, pero esto sería objeto de un futuro artículo.

El comportamiento equilibrado de las mujeres (40/60) no se corresponde con el de los varones, los cuales tienen tendencia a citar a sus homólogos masculinos (25/75). Ello da lugar a una situación negativa para las mujeres, pues si no reciben citas tienen problemas con su índice $h$ y sus méritos no son reconocidos por las agencias de acreditación y evaluación. Esto explica que la carrera universitaria de las mujeres siga un itinerario en el que ellas representan una mayoría en el comienzo de los estudios, una casi equiparación con los hombres cuando se alcanza el grado de doctor y un declive muy notable en el acceso al nivel de cátedra (LozanoAscencio et al., 2020), cátedras que, en 2020, en España, están ocupadas en el 80\% por hombres (Repiso et al., 2020).

De todas maneras, en relación con el éxito académico de las mujeres, hay que tener en cuenta también el hecho de que muchas mujeres entre 30 y 40 años abandonan la carrera para dedicarse a la maternidad, momentáneamente o ya para siempre (pues con la ausencia ya pierden el tren profesional) (Papí-Gálvez; Hernández-Ruiz; López-Berna, 2020). En España, en Comunicación, hay poca brecha de género en la autoría de las tesis doctorales, sin embargo, cuando se trata de dirigir proyectos de investigación I+D+i, la situación es muy desventajosa para las mujeres. Está bastante claro que no se siguen las recomendaciones de la Unión Europea en su programa Horizon 2020 cuyo primer objetivo es lograr el equilibrio de género en los equipos de investigación a todos los niveles (Lozano-Ascencio et al., 2020).

En la revisión bibliográfica ha quedado bastante patente que las obligaciones maternales de las mujeres son un handicap añadido para su ascenso en la carrera académica, lo cual debería tenerse en cuenta y compensarse de alguna manera, no solo para hacer justicia sino también para poder seguir contando con sus cualidades como investigadoras en las etapas senior.

Como han demostrado algunos estudios (Thelwall, 2020; Dion, Sumner y Mitchell, 2018), los patrones de género y citación varian según las disciplinas y las zonas geográficas. Una limitación de este estudio es que ha analizado el comportamiento de los autores sólo en el área de las Ciencias Sociales, y especialmente en Comunicación y Documentación.

Este estudio se ha limitado a analizar los autores que han publicado en 4 revistas del área de Ciencias Sociales que reciben artículos de autores de múltiples países, y no se ha tenido 
en cuenta su nacionalidad. Las cuatro revistas publican la gran mayoría de sus artículos en español, autorados por investigadores hispanoamericanos, lo cual es una limitación de nuestro estudio, y no lo hace extrapolable a otras naciones con culturas diferentes. En un futuro estudio más amplio, aunque bastante más laborioso, sería interesante observar los comportamientos de citación según la nacionalidad de las revistas y, en especial, de los autores.

Por último, declarar un deseo: nos gustaría que en una futura repetición de esta investigación se hallara un comportamiento más igualitario en cuanto a las citas a trabajos de mujeres y de hombres.

\section{Nota}

1. El efecto Matilda es un sesgo contra el reconocimiento de los logros de mujeres científicas cuyo trabajo se atribuye a sus colegas masculinos. Este efecto fue descrito por primera vez por la sufragista y abolicionista Matilda Joslyn Gage (1826-1898) en su ensayo "Woman as inventor" (publicado por primera vez como un tratado en 1870 y en la North American Review en 1883). El término "efecto Matilda" fue acuñado en 1993 por la historiadora de la ciencia Margaret W. Rossiter.

\section{Referencias bibliográficas}

Alvarado-López, María-Cruz; Martín-García, Noemí (2020). Techos de cristal en la industria publicitaria española: profesionales femeninas tras las campañas galardonadas en los premios a la eficacia de la comunicación comercial (2002-2018). El profesional de la información, 29 (3), España. (e290314). https://doi.org/10.3145/epi.2020.may.14

Andersen, Jens-Peter; Nielsen, Mathias-Wullum (2018). Google Scholar and Web of Science: Examining gender differences in citation coverage across five scientific disciplines. Journal of Informetrics, 12 (3), Holanda. (Pp. 950-959). https://doi.org/10.1016/j.joi.2018.07.010

Arzuaga-Williams, Mónica (2020). Rol del comunicador organizacional en Uruguay: tareas y funciones. Profesional de la información, 29 (3), España. (e290326). https://doi.org/10.3145/epi.2020.may.26

Azoulay, Pierre; Lynn, Freda B. (2020). SelfCitation, Cumulative Advantage, and Gender Inequality in Science. Sociological science, 7, Estados Unidos. (Pp. 152-186). https://doi.org/10.15195/v7.a7

Ballesteros-Herencia, Carlos A.; Gómez-García, Salvador (2020). Batalla de frames en la campaña electoral de abril de 2019. Engagement y promoción de mensajes de los partidos políticos en Facebook. Profesional de la información, 29(6), España. (e290629). https://doi.org/10.3145/epi.2020.nov.29

Beaudry, Catherine; Larivière, Vincent (2016). Which gender gap? Factors affecting researchers' scientific impact in science and medicine. Research Policy, 45(9), Holanda. (Pp. 17901817). https://doi.org/10.1016/i.respol.2016.05.009

Benjamens, Stan; Banning, Louise B. D.; Van-den-Berg, Tamar A. J.; Pol, Robert A. (2020). Gender Disparities in Authorships and Citations in Transplantation Research. Transplantation Direct, 6, Estados Unidos. (e614). https://doi.org/10.1097/TXD.0000000000001072 
Brown, Nadia E.; Samuels, David. (2018). Beyond the Gender Citation Gap: Comments on Dion, Sumner, and Mitchell. Political Analysis, 26 (3), Reino Unido. (Pp. 328-330). https://doi.org/10.1017/pan.2018.14

Budrikis, Zoe (2020). Growing citation gender gap. Nature Reviews Physics, 2, Reino Unido. (p. 346). https://doi.org/10.1038/s42254-020-0207-3

Correll, Shelley J. (2004). Constraints into Preferences: Gender, Status, and Emerging Career Aspiration. American Sociological Review 69 (1), Estados Unidos. (Pp. 93-113). https://doi.org/10.1177/000312240406900106

Dion, Michelle L.; Sumner, Jane-Lawrence; Mitchell, Sara-McLaughlin (2018). Gendered citation patterns across political science and social science methodology fields. Political analysis 26(3), Reino Unido. (Pp. 312-327). https://doi.org/10.1017/pan.2018.12

Dworkin, Jordan D.; Linn, Kristin A.; Teich, Erin G.; Zurn, Perry; Shinohara, Russell T.; Bassett, Danielle S. (2020). The extent and drivers of gender imbalance in neuroscience reference lists. Nature Neuroscience (23), Reino Unido. (Pp. 918-926). https://doi.org/10.1038/s41593-020-0658-y

Etura-Hernández, Dunia; Martín-Jiménez, Virginia; Ballesteros-Herencia, Carlos A. (2019). La comunidad universitaria, frente-la igualdad de género: un estudio cuantitativo. Revista Latina de Comunicación Social (74), España. (Pp. 1781-1800). http://doi.org/10.4185/RLCS-2019-1410

Exley, Christine L.; Kessler, Judd B. (2019). The gender gap in self-promotion. Working paper 26345. National Bureau of Economic Research. Estados Unidos. https://doi.org/10.3386/w26345

Fox, Mary-Frank; Whittington, Kjersten Bunker; Linkova, Marcela (2017). Gender, (In)equity, and the Scientific Workforce. Chapter 24. In: The Handbook of Science and Technology Studies, $4^{\text {th }}$ ed. Eds.: Ulrike Felt, Rayvon Fouche, Clark A. Miller, and Laurel SmithDoerr. Cambridge, Mass: MIT Press. Estados Unidos. https://www.researchgate.net/profile/Mary_Fox4/publication/292147982_Gender_InE quity and the Scientific Workforce/links/5a9a5621a6fdcc3cbac93d59/GenderInEquity-and-the-Scientific-Workforce.pdf

Fulvio, Jacqueline M.; Akinnola, Ileri; Postle, Bradley R. (2020). Gender (Im)balance in Citation Practices in Cognitive Neuroscience. Journal of Cognitive Neuroscience, 33 (1), Estados Unidos. (Pp. 3-7). https://doi.org/10.1162/jocn_a 01643

Ghiasi, Gita; Mongeon, Philippe; Sugimoto, Cassidy R.; Larivière, Vincent (2018). Gender homophily in citations. Proceedings of the 23rd Intl Conf on Science and Technology Indicators, Leiden, The Netherlands, (Pp. 1519-1525). https://scholarlypublications.universiteitleiden.nl/access/item\%3A2729532/view

González-Alcaide, Gregorio; González-Teruel, Aurora (2020). Dirección de tesis doctorales como reflejo de la Biblioteconomía y Documentación académica española: actores y temas. Profesional de la información, 29 (4), España. (e290441). https://doi.org/10.3145/epi.2020.jul.41

Herbst, Tessie H.H. (2020). Gender differences in self-perception accuracy: The confidence gap and women leaders' underrepresentation in academia. SA Journal of Industrial Psychology, 46, Sudáfrica. (a1704). https://doi.org/10.4102/sajip.v46i0.1704 
Holman, Luke; Stuart-Fox, Devi; Hauser, Cindy E. (2018). The gender gap in science: How long until women are equally represented? PLoS Biol, 16(4), Estados Unidos. (e2004956). https://doi.org/10.1371/journal.pbio.2004956

Huang, Mingqian; Naser-Tavakolian, Kiyon; Clifton, Michael; Franceschi, Ana M.; Kim, Derek; Zhang, Jill; Schweitzer, Mark (2019). Gender Differences in Article Citations by Authors from American Institutions in Major Radiology Journals. Cureus, 11 (8), Estados Unidos. (e5313). https://doi.org/10.7759/cureus.5313

Iranzo-Cabrera, María (2020). \#lasperiodistasparamos, gestación de una conciencia profesional feminista. El profesional de la información, 29 (2), España. (e290222). https://doi.org/10.3145/epi.2020.mar.22

King, Molly M.; Bergstrom, Carl T.; Correll, Shelley J.; Jacquet, Jennifer; West, Jevin D. (2017). Men set their own cites high: gender and self-citation across fields and over time. Socius: Sociological research for a dynamic world, 3, Estados Unidos. (online first). https://doi.org/10.1177/2378023117738903

Krawczyk, Michał (2017). Are all researchers male? Gender misattributions in citations. Scientometrics (110), Holanda. (Pp. 1397-1402). https://doi.org/10.1007/s11192-0162192-y

Larivière, Vincent; Ni, Chaoqun; Gingras, Yves; Cronin, Blaise; Sugimoto, Cassidy R. (2013). Global gender disparities in science. Nature, 504 (7479), Reino Unido. (Pp. 211-213). https://www.nature.com/news/bibliometrics-global-gender-disparities-in-science-

\subsection{1}

Lerchenmueller, Marc J.; Sorenson, Olav; Jena, Anupam B. (2019). Gender differences in how scientists present the importance of their research: observational study, BMJ, 367, Reino Unido. (16573). https://doi.org/10.1136/bmj.l6573

López-de-Ayala, María-Cruz; Vizcaíno-Laorga, Ricardo; Montes-Vozmediano, Manuel (2020). Hábitos y actitudes de los jóvenes ante las redes sociales: influencia del sexo, edad y clase social. Profesional de la información, 29 (6), España. (e290604). https://doi.org/10.3145/epi.2020.nov.04

Lozano-Ascencio, Carlos; Gaitán-Moya, Juan-Antonio; Caffarel-Serra, Carmen; Piñuel-Raigada, José-Luis (2020). Una década de investigación universitaria sobre Comunicación en España, 2007-2018. Profesional de la información, 29(4), España. (e290412). https://doi.org/10.3145/epi.2020.jul.12

Maliniak, Daniel; Powers, Ryan; Walter, Barbara F. (2013). The Gender Citation Gap in International Relations. International Organization, 67, Reino Unido. (Pp. 889-922). https://doi.org/10.1017/S0020818313000209

Mishra, Shubhanshu; Fegley, Brent D.; Diesner, Jana; Torvik, Vetle I. (2018). Self-citation is the hallmark of productive authors, of any gender. PLoS One, 13(9), Estados Unidos. (e0195773). https://doi.org/10.1371/journal.pone.0195773

Nielsen, Mathias Wullum (2017). Gender and citation impact in management research. Journal of Informetrics, $11 \quad(4), \quad$ Holanda. (Pp. 1213-1228). https://doi.org/10.1016/j.joi.2017.09.005

Nunkoo, Robin; Hall, C. Michael; Rughoobur-Seetah, Soujata; Teeroovengadum, Viraiyan (2019). Citation practices in tourism research: Toward a gender conscientious 
engagement. Annals of Tourism Research, 79, Reino Unido. (102755). https://doi.org/10.1016/j.annals.2019.102755

Papí-Gálvez, Natalia; Hernández-Ruiz, Alejandra; López-Berna, Sonia (2020). Digital transformation in the advertising industry from a gender perspective: an analysis of the top agencies. El profesional de la información, 29 (3), España. (e290323). https://doi.org/10.3145/epi.2020.may.23

Potthoff, Matthias; Zimmermann, Fabian (2017). Is there a gender-based fragmentation of communication science? An investigation of the reasons for the apparent gender homophily in citations. Scientometrics (112), Holanda. (Pp. 1047-1063). https://doi.org/10.1007/s11192-017-2392-0

Repiso, Rafael; Berlanga, Inmaculada; Said-Hung, Elías; Castillo-Esparcia, Antonio (2020). Titularidad y cátedras en Comunicación en España (2000-2019). Distribución, ritmos de promoción, transferencia entre universidades y endogamia. Profesional de la información, 29(4), España. (e290422). https://doi.org/10.3145/epi.2020.jul.22

Reuben, Ernesto; Sapienza, Paola; Zingales, Luigi (2014). How stereotypes impair women's careers in science. PNAS, 111(12), Estados Unidos. (Pp. 4403-4408). https://doi.org/10.1073/pnas.1314788111

Thelwall, Mike (2020). Gender differences in citation impact for 27 fields and six English-speaking countries 1996-2014. Quantitative science studies, 1(2), Estados Unidos. (Pp. 599617). https://doi.org/10.1162/qss_a 00038

Torres-Salinas, Daniel; Muñoz-Muñoz, Ana M.; Jiménez-Contreras, Evaristo (2011). Análisis bibliométrico de la situación de las mujeres investigadoras de Ciencias Sociales y Jurídicas en España. Revista española de documentación científica, 34(1), España. (Pp. 11-28). https://doi.org/10.3989/redc.2011.1.794 